Check for updates

Cite this: RSC Adv., 2017, 7, 41369

Received 8th June 2017

Accepted 8th August 2017

DOI: $10.1039 / c 7 r a 06429 b$

rsc.li/rsc-advances

\section{Reversible structural phase transition, ferroelectric and switchable dielectric properties of an adduct molecule of hexamethylenetetramine ferrocene carboxylic acid $\dagger$}

\author{
Yun-Zhi Tang, D* Yi Liu, Ji-Xing Gao, Chang-Feng Wang, Bin Wang, Yu-Hui Tan \\ and He-Rui Wen (D)
}

\begin{abstract}
Metallo-organic molecules showing reversible structural phase transitions accompanied with ferroelectric and dielectric properties have seldom been reported. By making use of the "driving/controlling force" from the rotating motion of cyclopentadienyl rings for a reversible structural transition and then carrying out structural modifications on their local parts to create remnant polarization through this molecular system, we designed and constructed a new ferroelectric, which is the adduct of hexamethylenetetramine ferrocene carboxylic acid, $\left(\mathrm{C}_{6} \mathrm{H}_{12} \mathrm{~N}_{4}\right)\left[\mathrm{Fe}\left(\mathrm{C}_{5} \mathrm{H}_{5}\right)\left(\mathrm{C}_{6} \mathrm{H}_{5} \mathrm{O}_{2}\right)\right]$ (1). Compound 1 undergoes a reversible structural phase transition at around $187 \mathrm{~K}$, which is confirmed by remarkable dielectric and heat anomalies. The synergistic interactions of the twisting motions of the adduct molecules and the inner cyclopentadienyl rings lead to the reversible phase transition. A dipole moment from amine group to carboxylic acid at low temperature produces a net spontaneous polarization, which results in the ferroelectricity of 1-LTP. On further investigation, it is observed that compound 1 has a perfect ferroelectric hysteresis loop with a spontaneous polarization of $3.95 \mu \mathrm{C} \mathrm{cm}^{-2}$.
\end{abstract}

\section{Introduction}

As smart materials, ferroelectrics have been widely utilized in various sensors and devices such as pyroelectric sensors, piezoelectric devices, high-permittivity dielectrics, electro-optic devices and positive temperature coefficient (PTC) of resistivity components. ${ }^{1-5}$ Recently, research on molecular ferroelectrics have attracted much interest since they have great advantages such as being lightweight, easy to prepare and environmentally friendly, in comparison with the conventional inorganic ferroelectric materials. ${ }^{6-13}$ Although a great progress has been made in the last few decades, few cases about metallo-organic molecular ferroelectrics have been documented. Moreover, the accurate design and construction of a molecular ferroelectric by facile structural modification on an existing metallo-organic molecule system is still a huge challenge. ${ }^{\mathbf{1 4 - 2 0}}$

In fact, a number of molecules that often undergo reversible phase transitions in the vicinity of the Curie temperature do not belong to the category of ferroelectrics, either above or below the phase transition temperatures. This is because

School of Metallurgy and Chemical Engineering, Jiangxi University of Science and Technology, Ganzhou, 341000, Jiangxi Province, P. R. China. E-mail: Tangyunzhi75@163.com

$\dagger$ Electronic supplementary information (ESI) available. CCDC 1548386 for 1-LTP $(110 \mathrm{~K}), 1548387$ for 1-HTP (300 K). For ESI and crystallographic data in CIF or other electronic format see DOI: $10.1039 / \mathrm{c} 7 \mathrm{ra06429b}$ a ferroelectric is required to satisfy some specific conditions: (i) their lower symmetrical structures must fall into one of the 10 noncentrosymmetric and polar space groups $(1,2, m, m m 2,3$, $3 \mathrm{~m}, 4,4 \mathrm{~mm}, 6$ and $6 \mathrm{~mm}$ ), ${ }^{21}$ and (ii) the dipole moments that can generate remnant spontaneous polarization should exist in the ferroelectric structures. ${ }^{22-28}$ However, in a molecular system with reversible phase transitions, if we fix a body part that can afford the "driving/controlling force" of the structural transition and carry out certain structural modifications to create polarization, can we design and construct a new ferroelectric through this molecular system?

Ferrocene, the first reported sandwich complex, possesses two typical reversible structures, the overlapping $\left(D_{5 \mathrm{~h}}\right)$ and interlaced $\left(D_{5 \mathrm{~d}}\right)$ cyclopentadienyl rings (Scheme 1). ${ }^{29-31}$ Since neither $D_{5 \mathrm{~h}}$ nor $D_{5 \mathrm{~d}}$ belong to polar space groups, they cannot be classified as ferroelectrics. However, in ferrocene, the twist (or rotate) motion of two cyclopentadienyl rings affords a strong "driving/controlling force" for a reversible structural transition. Ferrocene derivatives, such as adducts of ferrocene carboxylic acid with amines, may have acentric polar structures that could generate a permanent dipole moment from ferrocene carboxylic acid towards amine. Inspired by this idea, we prepared a series of adducts of ferrocene carboxylic acid with different amines. Fortunately, an adduct of hexamethylenetetramine ferrocene carboxylic acid, $\left(\mathrm{C}_{6} \mathrm{H}_{12} \mathrm{~N}_{4}\right)\left[\mathrm{Fe}\left(\mathrm{C}_{5} \mathrm{H}_{5}\right)\left(\mathrm{C}_{6} \mathrm{H}_{5} \mathrm{O}_{2}\right)\right]$ (1), was successfully fabricated and proven to be a molecular ferroelectric. To 

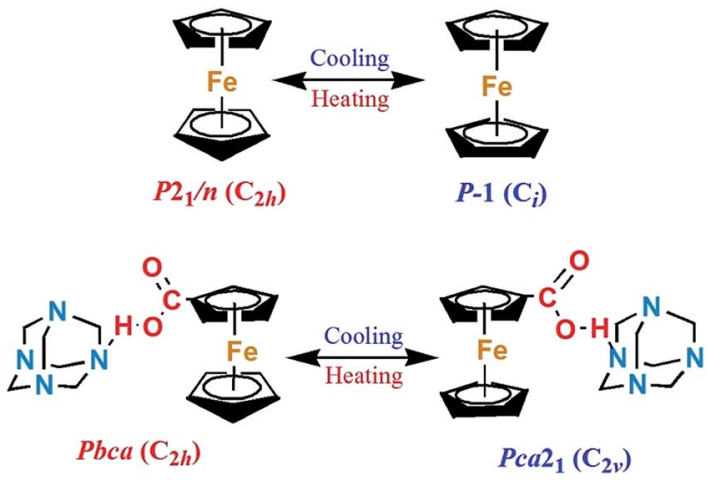

Scheme 1 Rotational motion of cyclopentadienyl rings triggers the reversible structural transitions.

the best of our knowledge, 1 represents the first metallo-organic compound that displays ferroelectric properties. Herein, we detailed its reversible structural phase transition, the mechanism of polarization, switchable heat and dielectric anomalies as well as the ferroelectric property.

\section{Experimental sections}

\subsection{Preparation of compound 1}

The 1:1 adduct of hexamethylenetetramine and ferrocene carboxylic acid $\left(\mathrm{C}_{6} \mathrm{H}_{12} \mathrm{~N}_{4}\right)\left[\mathrm{Fe}\left(\mathrm{C}_{5} \mathrm{H}_{5}\right)\left(\mathrm{C}_{6} \mathrm{H}_{5} \mathrm{O}_{2}\right)\right]$ (1) was prepared along with the $1: 2$ adduct of hexamethylenetetramine and ferrocene carboxylic acids $\left(\mathrm{C}_{6} \mathrm{H}_{14} \mathrm{~N}_{4}\right)\left[\mathrm{Fe}\left(\mathrm{C}_{5} \mathrm{H}_{5}\right)\left(\mathrm{C}_{6} \mathrm{H}_{4} \mathrm{O}_{2}\right)\right]_{2}$ (2) as per previously reported procedure. ${ }^{29,32}$ All reagents and solvents used in the reaction were purchased from commercially available sources. As shown in Scheme 2 and $1 \mathrm{mmol}(0.230 \mathrm{~g})$ ferrocene carboxylic acid and $1 \mathrm{mmol}(0.140 \mathrm{~g})$ hexamethylenetetramine were dissolved in $15 \mathrm{~mL}$ methanol separately and then mixed at $75{ }^{\circ} \mathrm{C}$ for 12 hours. The mixed orange crystals of $\mathbf{1}$ and $\mathbf{2}$ were formed after a few days of slow evaporation. The further experiment showed that the higher molar ratio of hexamethylenetetramine (HMTA) and ferrocene carboxylic acid (such as $3: 1$ ) is favorable of product 1 , resulting in a yield of about $69 \%$. We discerned that the crystal sample of 1 appeared orange and prismatic, while 2 was also orange but thin platelike. Analysis found for 1 at room temperature: C 55.4, $\mathrm{H} \mathrm{5.8,}$ $\mathrm{N} 15.1 \%$ and $\mathrm{C}_{17} \mathrm{H}_{22} \mathrm{FeN}_{4} \mathrm{O}_{2}$ required: C 55.1, H 5.9, N 15.0\%.

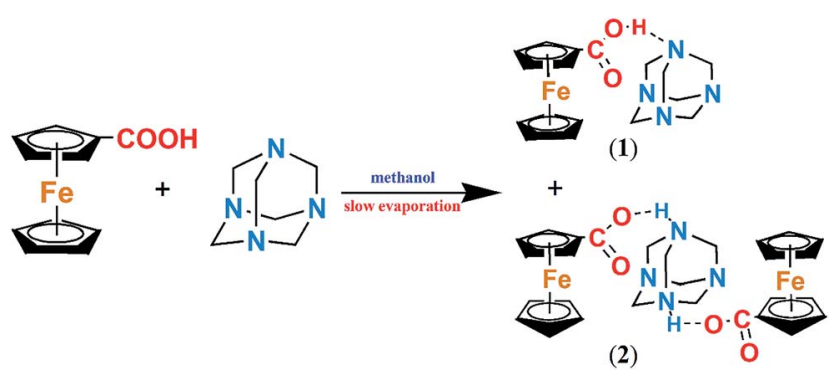

Scheme 2 The preparation of compound $\left(\mathrm{C}_{6} \mathrm{H}_{12} \mathrm{~N}_{4}\right)\left[\mathrm{Fe}\left(\mathrm{C}_{5} \mathrm{H}_{5}\right)\left(\mathrm{C}_{6} \mathrm{H}_{5}\right.\right.$ $\left.\left.\mathrm{O}_{2}\right)\right](1)$ and $\left(\mathrm{C}_{6} \mathrm{H}_{14} \mathrm{~N}_{4}\right)\left[\mathrm{Fe}\left(\mathrm{C}_{5} \mathrm{H}_{5}\right)\left(\mathrm{C}_{6} \mathrm{H}_{4} \mathrm{O}_{2}\right)\right]_{2}$ (2).
The crystals that were suitable for single-crystal X-ray diffraction were selected one by one directly from the analytical samples.

\subsection{Single-crystal X-ray crystallography}

$\mathrm{X}$-ray diffraction determinations for $\mathbf{1}$ were carried out above and below the phase transition temperature at 300 and $110 \mathrm{~K}$, respectively, through a Bruker Smart Apex II Single Crystal Diffractometer with Mo-K $\alpha$ radiation $(\lambda=0.71073 \AA)$. For convenience, we labeled the structure below the phase transition temperature $\left(T_{\mathrm{C}}=187 \mathrm{~K}\right)$ as 1-LTP and that above $T_{\mathrm{C}}$ as 1-HTP. All treatments including data collection, cell refinements, and data reduction were conducted using the CrystalClear Software package. ${ }^{33,34}$ The cell parameters and refinements of 1 at $300 \mathrm{~K}(\mathbf{1 - H T P})$ and $110 \mathrm{~K}(\mathbf{1 - L T P})$ are listed in Table 1. CCDC No. 1548386 for 1-LTP (110 K), 1548387 for 1-HTP $(300 \mathrm{~K})$.

\subsection{Measurement methods}

DSC measurements were conducted through a PerkinElmer Diamond DSC under a nitrogen atmosphere with a heating or cooling rate of $5 \mathrm{~K} \mathrm{~min}^{-1}$. The detailed analyses were carried out on a Quantum Design PPMS. Above room temperature, thermogravimetric analysis (TGA) measurements were performed using TA-Instruments STD 2960 from 293 to 900 K. Dielectric measurements were made with the pressed powder pellets of compound 1. Silver conduction paste was deposited on both surfaces of the pellet, which were used as the electrodes. Dielectric constants of $\mathbf{1}$ were determined with an Agilent or a TH2828A impedance analyser over a frequency range from $1 \mathrm{kHz}$ to $1 \mathrm{MHz}$. Ferroelectric hysteresis loops were measured from a powdered sample in the form of a pellet using a standard ferroelectric tester (RADIANT TECHNOLOGIES INC 609B) at $167 \mathrm{~K}$. Both surfaces of the pellet were coated with a silver conductive adhesive. The electric hysteresis loops were observed by virtual ground mode. The measurement was taken with alternating current and the frequency was 5-10 Hz.

\section{Results and discussions}

\subsection{Unit cell parameters differences between 1-RTP and 1- LTP}

As shown in Table 1, remarkable differences can be clearly observed between 1-RTP and 1-LTP. First, since the empirical formula of 1-LTP is twice that of 1-RTP, the asymmetrical unit of 1-RTP contains only one hexamethylenetetramine and one ferrocene carboxylic acid, while 1-LTP constitutes of two hexamethylenetetramines and two ferrocene carboxylic acids. Second, the unit cell length $a_{1 \text {-RTP }}$ and $c_{1 \text {-RTP }}$ are similar to $c_{1 \text {-LTP }}$ and $a_{1 \text {-LTP }}$, respectively, while the unit cell length $b$ remains unchanged. In addition, the unit cell volume also shrinks from $3268.5(5)$ to $3171.8(5) \AA^{3}$ that is about $2.96 \%$. Moreover, 1-RTP belongs to orthorhombic crystal system with centrosymmetrical Pbca (no. 61) space group, while 1-LTP crystallizes in an acentric $P c a 2_{1}$ (no. 29) space group. $P b c a$ has $D_{2 \mathrm{~h}}$ point group with eight symmetrical elements $\left(E, C_{2}, 2 C_{2}^{\prime}, i, \sigma_{\mathrm{h}}\right.$ and $2 \sigma_{\mathrm{v}}$ ), and $P c a 2_{1}$ belongs to $C_{2 \mathrm{v}}$ point group with four 
Table 1 Crystal data for 1-RTP (at $300 \mathrm{~K}$ ) and 1-LTP (110 K)

\begin{tabular}{lll}
\hline & 1-RTP & 1-LTP \\
\hline Empirical formula & $\mathrm{C}_{17} \mathrm{H}_{22} \mathrm{FeN}_{4} \mathrm{O}_{2}$ & $\mathrm{C}_{34} \mathrm{H}_{44} \mathrm{Fe}_{2} \mathrm{~N}_{8} \mathrm{O}_{4}$ \\
Formula weight & 370.24 & 740.47 \\
Temperature & $300(2) \mathrm{K}$ & $110(2) \mathrm{K}$ \\
Crystal system & Orthorhombic & Orthorhombic \\
Space group & $P b c a$ & $P c a 2_{1}$ \\
$a(\AA)$ & $19.883(2)$ & $22.958(2)$ \\
$b(\AA)$ & $7.1179(7)$ & $7.0548(6)$ \\
$c(\AA)$ & $23.095(2)$ & $19.5831(17)$ \\
$V\left(\AA^{3}\right)$ & $3268.5(5)$ & $3171.8(5)$ \\
$D_{\text {calca }} / \mathrm{Mg} \mathrm{m}^{-3}$ & 1.505 & 1.551 \\
$Z$ & 8 & 4 \\
$\mu\left(\mathrm{mm}{ }^{-1}\right)$ & 0.941 & 0.969 \\
$\mathrm{GOF}$ & 1.025 & 1.064 \\
$R_{1}[I>2 \sigma(I)]$ & $R_{1}=0.0796$, & $R_{1}=0.0335$, \\
& $\mathrm{w} R_{2}=0.1290$ & $\mathrm{w} R_{2}=0.0767$ \\
$\mathrm{w} R_{2}($ all data $)$ & $R_{1}=0.2015$, & $R_{1}=0.0354$, \\
$\Delta \rho_{\text {max }} / \Delta \rho_{\text {min }}\left(\mathrm{e} \AA^{-3}\right)$ & $\mathrm{w} R_{2}=0.1648$ & $\mathrm{w} R_{2}=0.0777$ \\
& $0.645 /-0.335$ & $0.525 /-0.247$ \\
& &
\end{tabular}

symmetrical elements $\left(E, C_{2}\right.$ and $\left.2 \sigma_{\mathrm{v}}\right)$. Clearly, the space group of 1-LTP $\left(\mathrm{Pca}_{1}\right)$ is the subgroup of 1-RTP $(\mathrm{Pbca})$. Therefore, it is observed that a symmetry breakdown occurs during phase transition. This is in good agreement with the principle of Aizu notation of $\mathrm{mmm}$, Fmm2 (Fig. 1). ${ }^{1-5}$

\subsection{Crystal structural differences between 1-RTP and 1-LTP}

As illustrated in Fig. 2a, the asymmetry unit of 1-RTP consists of one neutral ferrocene carboxylic acid and one hexamethylenetetramine adduct. All atoms of ferrocene carboxylic acid and hexamethylenetetramine are completely ordered. Two cyclopentadienyl rings are fully interlaced with each other and the Fe atom is located at their inversion center. Without exception, hexamethylenetetramine has a normal "diamond" structure, where all $\mathrm{N}$ atoms adopt $\mathrm{sp}^{3}$ hybrid orbital configuration and bond to different $\mathrm{C}$ atoms. Compared to 1-RTP, 1-LTP appears much different (Fig. 2b). The asymmetry unit of 1-LTP comprises two independent hexamethylenetetramine and ferrocene carboxylic acid neutral molecules. Peculiarly, the homologous cyclopentadienyl rings in one of the neutral molecules are parallel to each other with eclipsed conformation, while the other two relevant cyclopentadienyl rings in the other neutral molecule still retain the staggered fashion. In fact, two independent neutral molecules point out different directions (based on the carboxylic acid orientation) with roughly 50 degree torsion angle (Fig. S1†), implying that a part of the neutral molecules has accomplished the rotation motions. Besides, in 1-RTP, all intermolecular hydrogen bond distances between carboxylic acid and hexamethylenetetramine (O2-H2A $\cdots \mathrm{N} 1)$ are $2.675 \AA$, denoting a symmetry motion, while two different hydrogen bond distances, which are $2.639 \AA$ and $2.603 \AA$, exist in 1-LTP, displaying asymmetry motions. Thus it is found that a hydrogen shift has occurred between the two independent molecules.

\subsection{Symmetry break between 1-RTP and 1-LTP}

To further understand the microscopic mechanism of the reversible phase transition, we conducted a comprehensive structural analysis for both 1-RTP and 1-LTP. Compared to $C_{2 \mathrm{v}}$, $D_{2 \mathrm{~h}}$ contains two additional twofold axes $\left(2 C^{\prime}{ }_{2}\right)$, one $\sigma_{\mathrm{h}}$ mirror plane and one inversion center (i). The $\sigma_{\mathrm{h}}$ symmetrical operations can also be obtained by the symmetric rotation of the twofold axis $\left(2 C_{2}^{\prime}\right)$ according to symmetric operation principle. Therefore, we revealed the disappearance of two $C^{\prime}{ }_{2}$ axes and one inversion center (i) in 1-LTP.

As detailed in Fig. 3a, for 1-RTP whether from $b$-axis or $c$-axis, one can easily observe a twofold axis $\left(C_{2 y}, C_{2 z}\right)$ that equally divides the hexamethylenetetramine molecules. The similar symmetrical twofold axis $\left(C_{2 y}, C_{2 x}\right)$ can be discerned along the $a$ axis (Fig. S2 $\dagger$ ). Besides, the inversion center clearly lies in the heart of the hexamethylenetetramine molecules. To satisfy the demands of $D_{2 \mathrm{~h}}$ space group, the same symmetry operation has to be found in the ferrocene carboxylic acid molecules, since each adjacent ferrocene carboxylic acid molecule adopts an inverted arrangement. It is observed that an inversion center is clearly located in the middle of two neighbor Fe atoms. A twofold axis $C_{2 z}$ which equally splits two Fe atoms and parallels to the $c$-axis direction can also be obtained. Similarly, we discovered another twofold axis $\left(C_{2 y}\right.$ and $\left.C_{2 y}\right)$ in the structure.
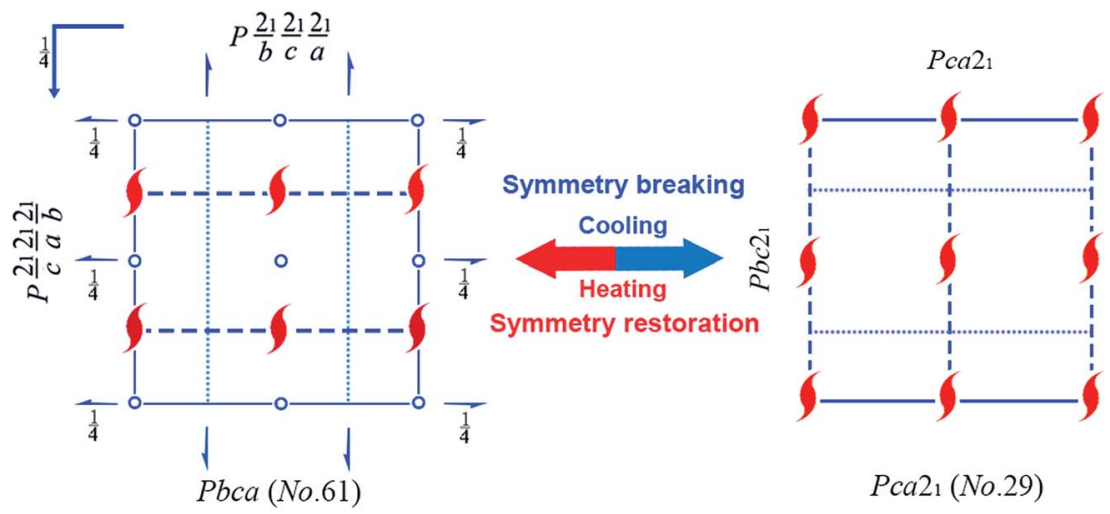

Fig. 1 The symmetry breaking between 1-RTP (left) and 1-LTP (right). 
a

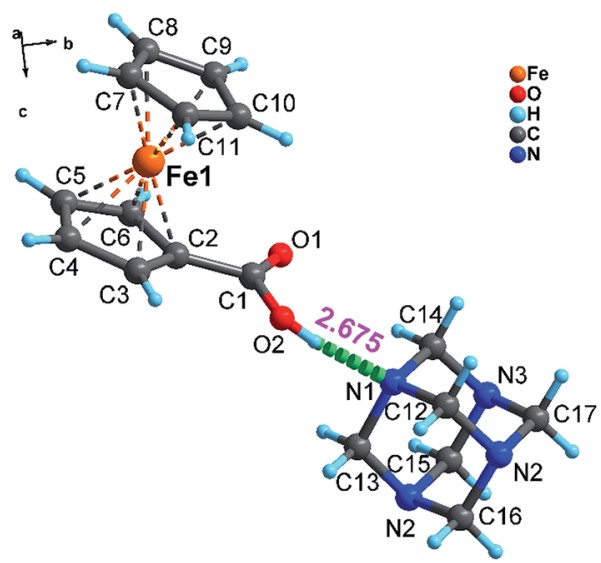

b

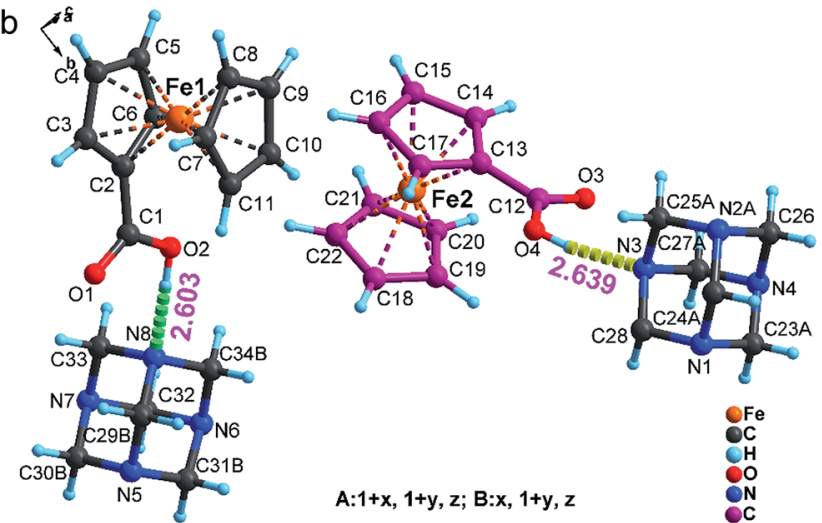

Fig. 2 (a) The asymmetry unit of 1-RTP with all the cyclopentadienyl rings adopt the same staggered conformation. (b) The asymmetry unit of 1-LTP shows the eclipsed conformation (highlighted in purple color) and staggered conformation (in grey color) of the cyclopentadienyl rings.

However, for 1-LTP (Fig. 3b), all adjacent ferrocene carboxylic acid molecules do not completely adopt antiparallel arrangements. Instead, they are intercrossed by about 50 degree (Fig. S1 $\dagger$ ). Thus, both symmetrical operations $\left(C_{2 x}\right.$ and $\left.C_{2 y}\right)$ are lost (Fig. S3†), while the $C_{2 z}$ symmetrical operation still exists (Fig. 3b). Furthermore, the cyclopentadienyl rings in both neutral molecules are not identical. Some parts of them adopt eclipsed conformation, while the others adopt staggered conformation. Therefore, the inversion center (i) vanishes in the middle of two neighbor Fe atoms. Overall, the synergistic interactions of the rotating motion between the independent adduct molecules and the inner cyclopentadienyl rings lead to the ultimate symmetrical breaking.

\subsection{Mechanism of polarization in 1-LTP}

Considering that 1-LTP belongs to one of the ten polar space groups ( $\mathrm{mm} 2$ ), there is a possibility that it is a molecular ferroelectric. As depicted in Fig. 3a and 4, we can define that a local dipole moment originates from the amine group toward carboxylic acid. In 1-RTP, the local dipole moments have opposite directions and cancel each other completely since all adjacent hexamethylenetetramine ferrocene carboxylic acids a

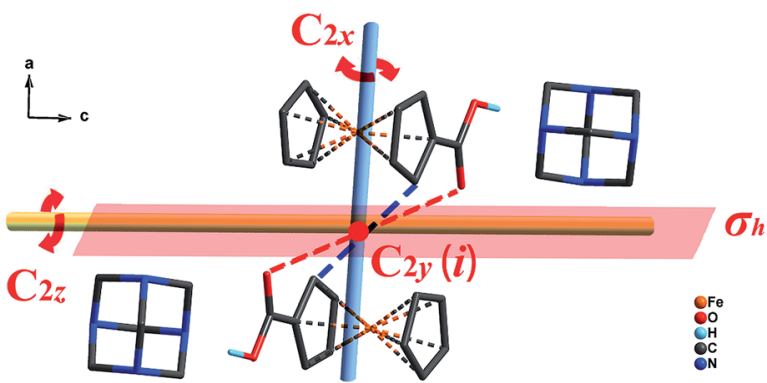

b

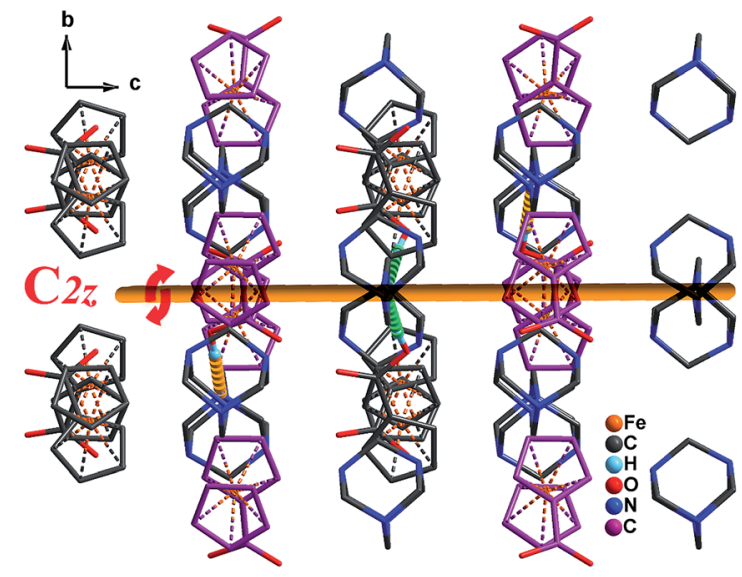

Fig. 3 (a) The packing structure of 1-RTP shows the symmetrical operations along the $b$-axis direction. (b) The packing structure of 1LTP shows the symmetrical operations viewed from a-axis direction.

adopt an inverted arrangement in an antiparallel fashion (Fig. 3a), and thus no net spontaneous polarization exists in the entire structure. In 1-LTP, a part of the hexamethylenetetramine ferrocene carboxylic acid molecule with staggered cyclopentadienyl rings rotates to a different direction (as highlighted in yellow color in Fig. 4). This part of neutral units still takes the inverted arrangement and does not produce net spontaneous polarization. However, the other part of hexamethylenetetramine ferrocene carboxylic acid molecules with eclipsed cyclopentadienyl rings exhibits a local dipole moment from carboxylic group toward amine. These dipole moment vectors along $a$-axis direction cannot cancel each other and thus superimpose over one another (as highlighted in red color in Fig. 4). As a result, the analogous dipole moment behaviors can be extended to the entire super molecules. A net spontaneous polarization is produced along the $a$-axis direction (highlighted in red color).

\subsection{Ferroelectric properties}

As expected, the thin pellet of compound $\mathbf{1}$ exhibits a perfect rectangular ferroelectric loop under $167 \mathrm{~K}$ (below its Curie temperature) with an applied electric potential of $600 \mathrm{~V}$, which affords a direct proof of the ferroelectric property (Fig. 5). The $P$ (polarization) $E$ (electric field) hysteresis loop shows that the saturated spontaneous polarizations are nearly equal to its remnant polarizations and always stabilize around $3.95 \mu \mathrm{C} \mathrm{cm}^{-2}$. To our knowledge, this value is slightly lower than that of 

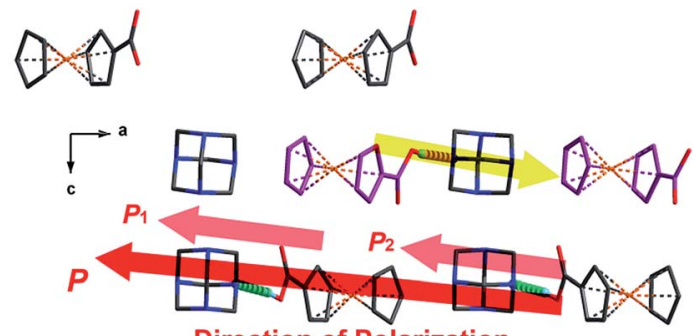

Direction of Polarization
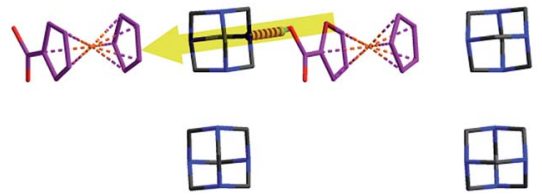

Fig. 4 The packing structure of 1-LTP shows the direction of spontaneous polarization.

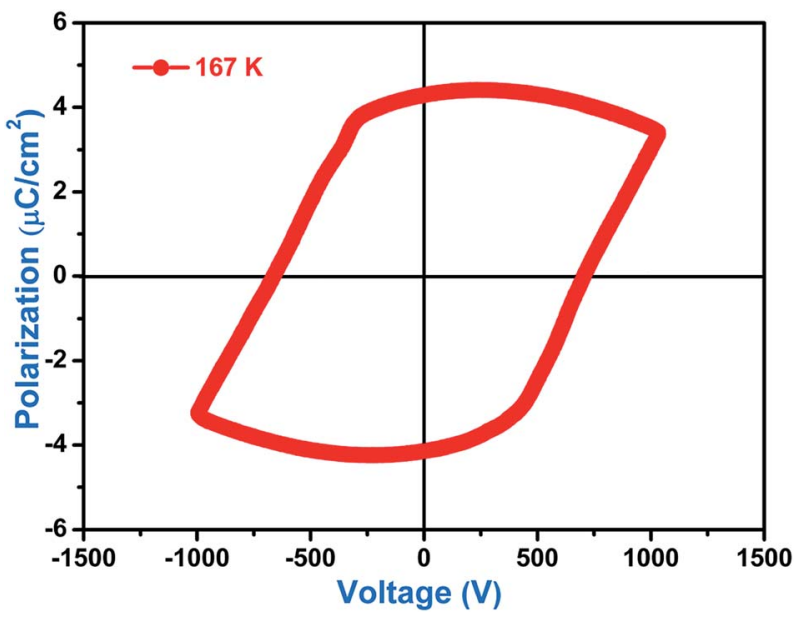

Fig. 5 Ferroelectric hysteresis loops measured from 1-LTP at $167 \mathrm{~K}$.

Rochelle salt $\left(\mathrm{NaKC}_{4} \mathrm{H}_{4} \mathrm{O}_{6} \cdot 4 \mathrm{H}_{2} \mathrm{O}\right)$, but much larger than that of most molecular ferroelectrics. In addition, this fashion of rectangular loops is similar to that of PZT (piezoelectric lead zirconate titanate), a classic ceramic ferroelectric. Since the pellet sample is fragile and should be handled with great care, no more detailed information was obtained.

\subsection{Dielectric properties}

The distinct changes of dielectric constants occurring in the vicinity of the phase transition are a significant criterion..$^{35}$ Thus, we carried out the measurements of the dielectric constants depending on temperature with the powder samples in a heating-cooling cycle mode. As shown in Fig. 6, the dielectric constant curve displays a striking inflection point at 192.3 $\mathrm{K}$ under frequency of $100 \mathrm{kHz}$ in the cooling run. The value of dielectric constant falls abruptly from 3.7 (a.u) to 3.2 (a.u), and then it decreases slowly with an decrease in temperature. Agreeing well with that of the cooling run, a steep increase of dielectric constant in the heating cycle starts at 182 $\mathrm{K}$ with 3.15 (a.u) and ends at $201 \mathrm{~K}$ with 3.55 (a.u). After that, the

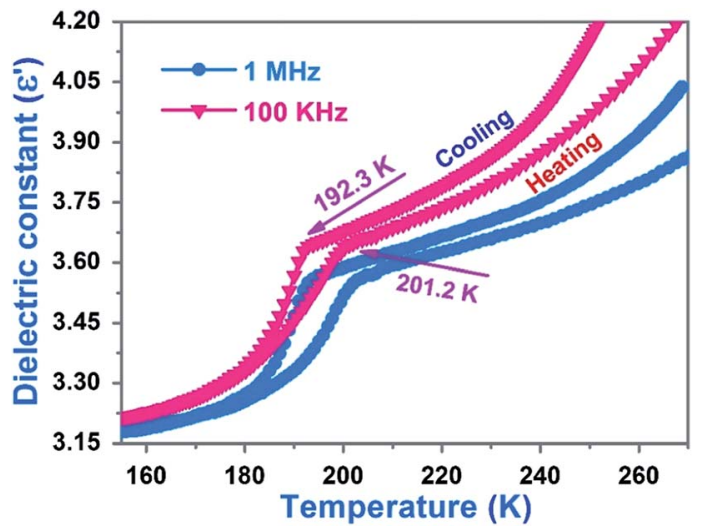

Fig. 6 The dielectric constant of 1 depending on temperature at frequency $1 \mathrm{MHz}$ and $100 \mathrm{kHz}$.

dielectric constant goes up slowly along a gentle slope with increase in temperature. The dielectric constant curve under 1 $\mathrm{MHz}$ frequency is similar to that under $100 \mathrm{kHz}$ except for a minor change in the value of the dielectric constant. The dielectric constants under $100 \mathrm{kHz}$ are higher than those under $1 \mathrm{MHz}$. This is well in accordance with the normal rule that the higher the frequency the lower the dielectric constant.

\subsection{Thermal properties}

In general, a reversible structural phase transition will display heat anomaly behavior in the vicinity of transition temperature. Therefore, we performed DSC measurements during heating and cooling process. As depicted in Fig. 7, the crystalline sample of 1 undergoes a remarkable phase transition at approximately $T_{\mathrm{C}}=187 \mathrm{~K}$ on the cooling and heating cycle, uncovering an exothermic peak at $187.0 \mathrm{~K}$ and an endothermic peak at $190.7 \mathrm{~K}$, which match well with the phase transition temperatures of dielectric anomalies of $\mathbf{1}$. Since both exothermic and endothermic peaks are very "steep" and have a large thermal hysteresis $(3.7 \mathrm{~K})$, we presume that this is possibly a first-order phase transition. Furthermore, we calculated the entropy changes $(\Delta S)$ for both cooling and heating cycle. The $\Delta S$ for the heating process is $1.866 \mathrm{~J} \mathrm{~mol}^{-1} \mathrm{~K}^{-1}$, calculated by using precise integration and calculus methods (ESI $\dagger$ ). Through the formula $\Delta H=T \Delta S(T=187.0 \mathrm{~K})$, one can deduce that the enthalpy change $(\Delta H)$ is about $339.6 \mathrm{~J} \mathrm{~mol}^{-1}$. Furthermore, from the $\Delta S=R \ln N$ (Boltzmann equation), where $R$ is the gas constant and $N$ represents the ratio of possible orientations, $N$ can be calculated as 1.252 at $187 \mathrm{~K}$. Similarly, the calculated entropy change $\left(\Delta S=1.790 \mathrm{~J} \mathrm{~mol}^{-1} \mathrm{~K}^{-1}\right)$ and $N$ value $(\approx 1.240)$ for the cooling process $(\approx 190 \mathrm{~K})$ were calculated $(\mathrm{ESI} \dagger)$, which are closer to those of heating process. ${ }^{1-5}$ Such a low activation energy of only a few $\mathrm{kJ} \mathrm{mol}^{-1}$ on changing structural conformation is very useful for a reversible phase transition material since it could sensitively respond to the thermal stimuli. To achieve further information about the stability of compound $\mathbf{1}$, we conducted TG-DTA experiments above room temperature. According to the TG-DTA curves from Fig. S4, $\dagger$ the decomposition temperature of 1 starts at $408.6 \mathrm{~K}$, much higher than the reversible phase transition temperature $187 \mathrm{~K}$. 


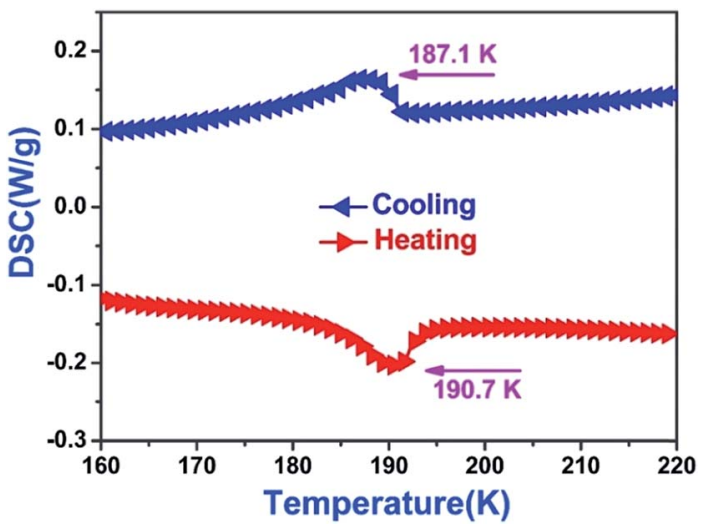

Fig. 7 DSC curves of 1 in the heating-cooling cycle showing heat anomaly around $187 \mathrm{~K}$.

\section{Conclusions}

In conclusion, using the "driving/controlling force" for a reversible structural transition in ferrocene molecular system, we have successfully designed and constructed an unprecedented metallo-organic ferroelectric, the adduct of hexamethylenetetramine ferrocene carboxylic acid, $\left(\mathrm{C}_{6} \mathrm{H}_{12} \mathrm{~N}_{4}\right)$ $\left[\mathrm{Fe}\left(\mathrm{C}_{5} \mathrm{H}_{5}\right)\left(\mathrm{C}_{6} \mathrm{H}_{5} \mathrm{O}_{2}\right)\right]$ (1). The synergetic rotating (twist) motions of the intermolecular and inner relevant cyclopentadienyl rings induce phase transition and lead to spontaneous polarizations. This investigation will provide a possible new venue to explore molecular ferroelectrics.

\section{Conflicts of interest}

There are no conflicts to declare.

\section{Acknowledgements}

Dr Tang is grateful for the support from the National Natural Science Foundation of China (Grant No. 21671086, No. 21261009, No. 21461010, No. 21471070), Jiangxi Province Science and Technology Support Program (20133BBE50020), and the Patent Sustentation Fund from Jiangxi Province (3203304622).

\section{Notes and references}

1 M. E. Lines and A. M. Glass, Principles and Application of Ferroelectrics and Related Materials, Oxford University Press, Clarendon, U. K., 1977.

2 U. Kenji, Ferroelectric Devices, Chemical Rubber Company Press, U. S., 2010.

3 T. Hang, W. Zhang, H. Y. Ye and R. G. Xiong, Chem. Soc. Rev., 2011, 40, 3577-3598.

4 W. Zhang, H. Y. Ye and R. G. Xiong, Coord. Chem. Rev., 2009, 253, 2980-2997.

5 P. P. Shi, Y. Y. Tang, P. F. Li, W. Q. Liao, Z. X. Wang, Q. Ye and R. G. Xiong, Chem. Soc. Rev., 2016, 45, 3811-3827.
6 G. Kieslich, A. C. Forse, S. Sun, K. T. Butler, S. Kumagai, Y. Wu, M. R. Warren, A. Walsh, C. P. Grey and A. K. Cheetham, Chem. Mater., 2016, 28, 312-317.

7 H. Y. Ye, W. Q. Liao, C. L. Hu, Y. Zhang, Y. M. You, J. G. Mao, P. F. Li and R. G. Xiong, Adv. Mater., 2016, 28, 2579-2586.

8 Z. H. Sun, X. T. Liu, T. Khan, C. M. Ji, M. A. Asghar, S. G. Zhao, L. N. Li, M. C. Hong and J. H. Luo, Angew. Chem., Int. Ed., 2016, 55, 6545-6550.

9 Z. H. Sun, T. L. Chen, J. H. Luo and M. C. Hong, Angew. Chem., Int. Ed., 2012, 51, 3871-3876.

10 Z. H. Sun, J. H. Luo, S. Q. Zhang, C. M. Ji, L. Zhou, S. H. Li, F. Deng and M. C. Hong, Adv. Mater., 2013, 25, 4159-4163.

11 D. W. Fu, W. Zhang, H. L. Cai, Y. Zhang, J. Z. Ge, R. G. Xiong and S. D. Huang, J. Am. Chem. Soc., 2011, 133, 12780-12786.

12 H. Y. Ye, S. H. Li, Y. Zhang, L. Zhou, F. Deng and R. G. Xiong, J. Am. Chem. Soc., 2014, 136, 10033-10040.

13 W. Zhang, H. Y. Ye, R. Graf, H. W. Spiess, Y. F. Yao, R. Q. Zhu and R. G. Xiong, J. Am. Chem. Soc., 2013, 135, 5230-5233.

14 H. Y. Ye, Y. Zhang, D. W. Fu and R. G. Xiong, Angew. Chem., Int. Ed., 2014, 126, 6724-6729.

15 Y. Zhang, H. Y. Ye, D. W. Fu and R. G. Xiong, Angew. Chem., Int. Ed., 2014, 53, 2114-2118.

16 Z. Sun, T. Chen, C. Ji, S. Zhang, S. Zhao, M. Hong and J. Luo, Chem. Mater., 2015, 27, 4493-4498.

17 S. Zhao, P. Gong, S. Luo, L. Bai, Z. Lin, C. Ji, T. Chen, M. Hong and J. Luo, J. Am. Chem. Soc., 2014, 136, 8560-8563.

18 H. L. Cai, W. Zhang, J. Z. Ge, Y. Zhang, K. Awaga, T. Nakamura and R. G. Xiong, Phys. Rev. Lett., 2011, 107, 147601.

19 Y. Zhang, W. Q. Liao, D. W. Fu, H. Y. Ye, Z. N. Chen and R. G. Xiong, J. Am. Chem. Soc., 2015, 137, 4928-4931.

20 H. Y. Ye, Q. H. Zhou, X. H. Niu, W. Q. Liao, D. W. Fu, Y. Zhang, Y. M. You, J. Wang, Z. N. Chen and R. G. Xiong, J. Am. Chem. Soc., 2015, 137, 13148-13154.

21 Y. Z. Tang, Y. M. Yu, J. B. Xiong, Y. H. Tan and H. R. Wen, J. Am. Chem. Soc., 2015, 137, 13345-13351.

22 S. Horiuchi and Y. Tokura, Nat. Mater., 2008, 7, 357-366.

23 Z. H. Sun, Y. Y. Tang, S. Q. Zhang, C. M. Ji, T. L. Chen, M. C. Hong and J. H. Luo, Adv. Mater., 2015, 27, 4795-4801. 24 J. Z. Ge, X. Q. Fu, T. Hang, Q. Ye and R. G. Xiong, Cryst. Growth Des., 2010, 10, 3632-3637.

25 P. Zhou, Z. Sun, S. Zhang, C. Ji, S. Zhao, R. G. Xiong and J. Luo, J. Mater. Chem. C, 2014, 2, 2341-2345.

26 Z. Sun, T. Chen, X. Liu, M. Hong and J. Luo, J. Am. Chem. Soc., 2015, 137, 15660-15663.

27 S. Zhao, P. Gong, S. Luo, S. Liu, L. Li, M. A. Asghar, T. Khan, M. Hong, Z. Lin and J. Luo, J. Am. Chem. Soc., 2015, 137, 2207-2210.

28 Y. Z. Tang, Z. F. Gu, J. B. Xiong, J. X. Gao, Y. Liu, B. Wang, Y. H. Tan and Q. Xu, Chem. Mater., 2016, 28, 4476-4482.

29 S. Paul and D. D. Jack, Acta Crystallogr., Sect. B: Struct. Crystallogr. Cryst. Chem., 1979, 35, 2020-2023.

30 G. Galvarin, G. Clec'h and J. F. Berar, J. Phys. Chem. Solids, 1982, 43, 785-790.

31 L. Lin, A. Berces and H.-B. Kraatz, J. Organomet. Chem., 1998, 556, 11-20. 
32 M. Z. Choudhury, F. George, J. L. Alan and G. Christopher, Acta Crystallogr., Sect. C: Cryst. Struct. Commun., 2003, 59, $\mathrm{m} 271-\mathrm{m} 274$.

33 P. Muller, R. Herbst-Irmer, A. Spek, T. Schneider and M. Sawaya, Crystal Structure Refinement-A Crystallographer's Guide to SHELXL, Oxford University Press, New York, 2006.
34 G. M. Sheldrick, SHELXS-97, Programs zur Lösung vonKristallstrukturen, University of Göttingen, Göttingen, Germany, 1997.

35 W. J. Xu, C. T. He, C. M. Ji, S. L. Chen, R. K. Huang, R. B. Lin, W. Xue, J. H. Luo, W. X. Zhang and X. M. Chen, Adv. Mater., 2016, 28, 5886-5890. 